\title{
Avaliação da atenção à saúde da criança (0-5 anos) no PSF de Teresópolis (RJ) segundo a percepção dos usuários
}

\author{
Children health care evaluation ( $0-5$ years) according to \\ users' perceptions in the Family Health Strategy of Teresópolis, \\ Rio de Janeiro State
}

José $M$ endes Ribeiro ${ }^{1}$

Sandra A parecida Venâncio de Siqueira ${ }^{1}$

Luiz Felipe da Silva Pinto ${ }^{2}$

\footnotetext{
${ }^{1}$ Departamento deCiências Sociais, Escola Nacional de Saúde Pública Sérgio Arouca, Fundação Oswaldo Cruz. Rua Leopoldo Bulhões 1480/920, M anguinhos. 21045-210 Rio de Janeiro RJ. ribeiro@ensp.fiocruz.br ${ }^{2}$ Secretaria M unicipal de Saúde e D efesa Civil do Rio deJaneiro.
}

Abstract Family Health Strategy represents one of the major public programs in Brazil covering 5,601 municipalities in 2006 (91.8\%). This study evaluated Teresópolis Family Health Strategy through information and perceptions of adults responsible for children around 0-5 years that used those health services. It was used evaluation forms answered by family in waiting rooms or during home visiting. Results for urban and rural areas were compared and significant differences were observed in some issues. $M$ ajor findings include users' strong ties with the program and a highly positive perception about the children health status. Access was satisfactory as to use criteria when relating to regular care proceedings. Critical points observed include: low coverage of home visiting, bad access to labs and image exams and to systematic delivery of medicines. Rural areas presented a high number of children who treated acute clinical conditions at home without doctor assistance. U sers satisfaction as to physicians, nurses, health community agents and services were high level. U sers not regularly visited at home presented lower access to medicine delivery.

Key words Unified Health System, Family $\mathrm{H}$ ealth Strategy, Health services evaluation
Resumo O PSF representa um dos principaisprogramas públicos em saúde no Brasil, cobrindo em 2006 um total de 5.601 municípios (91,8\%). 0 estudo avaliou o PSF do município de Teresópolis (RJ) por meio da percepção e das informações prestadas pelos responsáveis por crianças na faixa de 0-5 anos e que utilizam estes serviços, por meio de questionários aplicados aos responsáveis nos próprios módulos de atendimento ou em visitas domiciliares. Resultados foram comparados entre áreas urbanas e rurais e diferenças significativas foram observadas para determinadositens eelevado vínculo da clientela ao programa euma percepção predominantemente positiva sobre o estado de saúde das crianças. 0 acesso se mostrou adequado segundo os critérios utilizados quando se refere ao atendimento de rotina. Os pontos críticos observados incluem baixa cobertura de visitas domiciliares, dificuldades na realização de exames solicitados eno fornecimento regular de medicamentos. As áreas rurais apresentaram um número elevado decriançasquetrataram condições agu das em casa sem assistência médica. A satisfação manifestada pel os usuários quanto aos médicos, aos agentes de saúde, aos profissionais de enfermagem e ao conjunto dos serviços foi elevada. A clientela não visitada por agentes desaúdeapresentou menor acesso à distribuição de medicamentos. Palavras-chave SUS, Programa de Saúde da Família, Avaliação em Saúde 
Introdução

O Sistema Ú nico de Saúde (SUS), institucionalmentedefinido por seu caráter público, universal e integral, financiado por recursos fiscais, apresentou ao longo da década de noventa um amplo rol de iniciativas orientadas a cumprir com suas disposições constitucionais. A necessidadedeprover 0 acesso a serviços de saúde para amplos contingentes populacionais em distintas regiões influenciou diferentes governos a desenvolver incentivos financeiros para a ampliação da oferta de serviços não-hospitalares. M odelos simplificados em termos do uso de profissionais de saúde, instalações, equipamento e meios diagnósticos eterapêuticosforam desenvolvidos em vários municípios brasileiros eal gumas experiências ganharam relevância em ambientes de al ta escassez de recursos e de pobreza. I gualmente em centros urbanos, experiências de instalações simplificadas e adaptáveis a áreas de difícil acesso e de risco social foram se multiplicando. Em comum, estas soluções tinham o foco em (i) grupos sociais altamente vulneráveis e com problemas de acesso a serviços de saúde; e (ii) reunir um número reduzido de profissionais de saúde seguindo a lógica de equipes sanitárias integradas.

Estes programas se tornaram nacionais, disseminados em áreas urbanas e rurais e, mais re centemente, passaram a assumir um caráter substitutivo com relação à rede tradicional de atenção primária. Este conjunto de experiências levou à unificação do conceito de atenção básica e à normatização de procedimentos de incentivos financeiros por partedo M inistério da Saúde por meio desucessivas $\mathrm{N}$ ormas $\mathrm{O}$ peracionais. Como resultado, a política oficial do SU S para o atendimento ambulatorial foi paulatinamente se concentrando na Atenção Básica ( $A B)$ e na Estraté gia de Saúde da Família (ESF), tendo sido sistematizada em 2006 mediante a Política Nacional de Atenção Básica (PNAB) ${ }^{1}$.

Para o M inistério da Saúde, a atenção básica envolve ações de promoção e proteção a agravos, desenvolvidas por equipes multidisciplinares orientadas a resolver os problemas de saúde de maior prevalência e incidência na região. $A$ estratégia de saúde da família é considerada sua principal ação no âmbito do sistema público.

A magnitude destes programas pode ser medida pelos gastos realizados pelo M inistério da Saúde no ano de 2005 para o financiamento de ESF, no total de $R \$ 2.679,27$ milhões. Para 0 ano de 2006, segundo o M inistério da Saúde, existia em todo o país um total de 219.492 agentes co- munitários de saúde; 26.729 equipes de saúde da família e 15.086 equipes de saúde bucal. Muitas destas equipes e agentes comunitários se superpõem nas mesmas instalações. 0 programa está presente em 5.601 municípios brasileiros (91,8\% do total).

A literatura sobre atenção básica e estratégia de saúde da família no Brasil foi marcada durante longo tempo por estudos de caso, propostas organizacionais e de estrutura de políticas ou difusão de casos supostamente exitosos, mesmo que desacompanhados de evidências mais sólidas de sucesso. Este tipo de produção refletiu, no entanto, o forte interesse do tema nos meios políticos, governamentais e acadêmicos. Teses desenvolvidas em programas de pós-graduação foram orientadas com muita frequência para estudos de caso de experiências conhecidas em diferentes cidades.

M ais recentemente, estudos têm buscado realizar amostras maiores, probabilísticas ou não, promover modelos de avaliação mais articulados aos observados em outros países e apresentar estudos comparativos entre a ESF e serviços ambulatoriais mais convencionais. Do ponto de vista metodológico, foram difundidas técnicas de estabelecimentos de linha de base, o que possibilitará a construção de séries históricas e futuras comparações dos efeitos obtidos com a implementação da política e a sustentação de sistemas demonitoramento evalidação de resultados mais permanentes ${ }^{2}$.

A despeito da controversa sobre o seu uso, outros estudos utilizam traçadores e eventos sentinelas, baseados em problemas de saúde da população, para avaliar a qualidade dos serviços em diversos países ${ }^{3-5}$. 0 pressuposto é de que 0 modo como as equipes médicas administram a atenção a doenças comuns indica a qualidade geral do sistema $a^{6,7}$. A seleção de traçadores deve contemplar características que favoreçam a sua especificidade, tais como impacto perceptível, critérios objetivos de diagnóstico, prevalência elevada, história natural sensível à intervenção mé dica, protocolos médicos bem definidos de intervenção e efeitos conhecidos defatores não mé dicos sobre o traçador escolhido. No caso brasileiro, os estudos mostram que indicadores diferentes foram utilizados como eventos sentinelas (taxa de mortalidade infantil, índice de mortes evitáveis, etc.) $)^{8}$.

Um ponto comum observado nas distintas abordagens está no fato de determinadas condições ou programas serem passíveis, embora limitadamente, de sinalizar, monitorar ou eviden- 
ciar aspectos substantivos da qualidade da atenção de serviços de saúde. Por outro lado, matrizes de análise mais abrangentes são destacadas para a avaliação da qualidade de serviços de saúde. Os componentes da qualidade contemplados em um modelo integrado de avaliação de políticas envolve: eficácia (influência de inovações tecnológicas); efetividade (grau de aproximação aos aprimoramentos possíveis); eficiência (economia de custos sem prejuízo de metas); otimização (equilíbrios entre custos e ganhos em condições desaúde); aceitabilidade (conformidadea expectativas dos usuários); legitimidade (conformidadeàs preferências sociais easpectos institucionais); e equidade (conformidade a princípios distributivos aceitos) ${ }^{9}$.

Embora a tomada de decisões seja dependente de aspectos subjetivos como, por exemplo, a credibilidade das equipes e consistências dos estudos realizados, a difusão de uma cultura avaliativa no país ${ }^{10}$ tem contribuído para que os centros colaboradores embasem as decisões do M inistério da Saúde (MS) ${ }^{11}$. Para tal, estes estudos têm procurado comparar o desempenho do programa em diversos aspectos: entre municípios de diversos portes, entre áreas cobertas e não cobertas, bem como analisado a aplicabilidade de model os desenvolvidos em outros países para a realidade brasileira ${ }^{12-14}$.

As análises trazem evidências que precisam ser mais bem compreendidas para uma avaliação sobre o desempenho do PSF: 1) não há consenso sobre a vantagem na qualidade do atendimento do PSF em relação à U BS entre os usuários, profissionais de saúde e gestores, a não ser em al guns indicadores deacesso, enfoquefamiliar e orientação para a comunidade; 2) evidência de pior cobertura e qualidade de atenção em municípios pequenos; 3) maior desigualdade de acesso onde a necessidade de cuidado é maior, balizada pelo poder de compra do usuário ${ }^{15-18}$.

Ainda nesta linha de estudos comparados, a literatura tem dado especial atenção às questões relativas às condições de trabalho dos profissionais. A precarização das relações de trabalho, as contratações por critérios políticos e o não cumprimento da carga horária emergem como problemas recorrentes ${ }^{19}$. Por fim, no tocante ao financiamento ao PSF, a literatura tem destacado que os incentivos lineares adotados pel os governos federal e estaduais para incentivar a implementação de grande número de equipes nos municípios não tem sido uma estratégia adequada em decorrência das características locais diferenciadas dos municípios, recaindo, em muitos casos, o ônus financeiro da implementação do PSF sobre os municípios ${ }^{20,21}$.

\section{Procedimentos metodológicos}

Em nosso estudo, a atenção à criança foi escolhida para avaliar o desempenho de módulos de saúde da família pelo fato de apresentar um conjunto de práticas que podem refletir o desempenho de áreas interdependentes da atenção a saúde, como a organização da porta de entrada, 0 sistema de agendamento de consultas, a qualidadee 0 acesso a serviços de laboratório e outros meios diagnósticos. 0 contato das crianças com o serviço de saúde é realizado por meio de seus responsáveis e, como amplamente conhecido, envolve no geral consultas de rotina para acompanhamento de desenvolvimento, visitas domiciliares de rotina por agentes de saúde e consultas médicas por problemas de saúde imediatos ou emergenciais.

A utilização de grupos sociais como fonte de informação para avaliar a qualidade de serviços tem sido feita em estudos nacionais, assim como as fronteiras metodológicas (limites efacilitação) quanto ao seu uso como traçadores. 0 foco na atenção básica se deve a fundamentos tradicionais da saúde pública que enfatizam a prevenção primária e a agenda da promoção da saúde ${ }^{22}$. A programação em saúde e a relação entre protocolos de atenção e estruturas organizacionais de atenção à saúde, nas quais programas de atenção à saúde da criança despertam especial interesse, também éfoco tradicional no setor ${ }^{23}$. Comparada à medicina subespecializada, a atenção primária seria menos intensiva tanto em capital, quanto em trabalho esua estrutura organizacional seria menos hierarquizada, favorecendo a adaptação a condições enecessidades locais. N este caso, a noção de atenção primária éabrangente e inclui as próprias estruturas de atenção gerenciada mais conhecidas (managed care). Considerando a tendência observada à convergência de sistemas nacionais de saúde, um elemento a ser levado em conta é que um sistema de saúde orientado à subespecialização ameaça os objetivos gerais de equidade ${ }^{24}$.

0 grupo de crianças na faixa etária de 0-5 anos foi utilizado como referência para avaliar a qualidade do PSF de Teresópolis segundo a percepção de seus usuários. Estetipo deestudo deve ser combinado a outras estratégias de investigação, como as consultas a bases administrativas e bancos de dados sobre oferta e produção de ser- 
viços. No entanto, é relevante para capturar de modo sistemático e analítico os pontos de vista dos usuários de serviços de saúde. A percepção dos usuários ganha importância específica no caso brasileiro, em que a tradição sanitarista enfatiza em demasia metodologias indiretas de definição de necessidades em saúde e de qualidade de serviços por meio de estimativas à distância dos serviços.

A rede de atenção primária é composta por estabelecimentos de saúde sem internação, centros de saúde, postos de saúde e unidades do Programa de Saúdeda Família. No município de Teresópolis, existiam, em 2004, dez Unidades de Saúde deFamília, que cobriam $28,25 \%$ da população, percentual menor do que o da região serrana, onde está localizado. N este ano, o percentual de crianças menores de cinco anos encontradas no município foi equivalente ao verificado nas dez áreas cobertas pelas Equipes de Saúde da Família e entre as áreas urbanas e rurais que possuem PSF, perfazendo um total de 12.902 crianças, ou seja 8,9\% dos 145.123 habitantes.

0 objetivo do delineamento amostral foi selecionar duas amostras independentes, uma para a área urbana (que considera as oito unidades de saúde existentes - Beira Linha, Araras, Rosário, Fonte Santa, Granja Florestal, Granja Guarani, M eudom e Barra do I mbuí) e outra para a área rural (Venda Nova e Vargem Grande), contemplando, ao final, todas as Unidades de Saúde da Família existentes. Essas duas amostras foram comparadas e as diferenças observadas testadas quanto às significâncias estatísticas como amostras al eatórias independentes. Para o cálculo do tamanho da amostra total, foi considerado o caso mais desfavorável onde a estimativa é de um parâmetro $(p)=q=0,5$, isto é, onde cada característica a ser investigada apresentaria a proporção observada de $50 \%$. Com isso, o tamanho total da amostra para um nível de confiança de $95 \%$ e com precisão esperada de suas estimativas (totais e proporções) na ordem de $4,5 \%$ foi de 456 pessoas para a área urbana e 395 para a área rural, al ocadas de forma proporcional a cada uma das dez áreas cobertas pelo Programa de Saúde da Família. Após a coleta dos dados, a amostra das dez unidades de saúde da área urbana foi de 381 pessoas ( $83,6 \%$ do total) entre as áreas cobertas pelas oito unidades de saúde da área urbana e 213 (53,9\% do total) entre as duas unidades de saúde da área rural, totalizando 594 entrevistas. Com isso, o erro de amostragem observado para as estimativas realizadas (para $p=q=0,5$ ) foi ajustado para $4,9 \%$ nas áreas ur- banas e $6,4 \%$ nas áreas rurais. Cerca de $80 \%$ do total das entrevistas foram realizadas no domicílio e $20 \%$ na própria unidade de saúde. A fórmula utilizada para a determinação do tamanho de cada uma das amostras aleatórias simples (uma para a área urbana e outra para a área rural) foi:

$$
n=\frac{N z^{2} S^{2}}{N d^{2}+z^{2} S^{2}} \text {, onde }
$$

N é o tamanho da população;

z éo correspondentena curva normal à con-

fiabilidade desejada;

S é o desvio padrão da população;

$S^{2}$ é a variância populacional;

d é a precisão desejada.

No caso de proporções, a variância da população toma a forma:

$$
\mathrm{S}^{2}=\frac{\mathrm{NPQ}}{\mathrm{N}-1}
$$

0 estudo, predominantementedomiciliar, foi facilitado pela existência e apoio dos agentes comunitários de saúde da Estratégia de Saúde da Família. Além disso, foi fundamental para o trabalho a existência do cadastro familiar - onde, através da Ficha $A$, os dados das pessoas são coletados e atualizados -, o que possibilitou a identificação das pessoas amostradas pelos domicílios. Para os entrevistados nas unidades, 0 critério de seleção foi a inclusão detodas as crianças de 0-5 anos presentes para consulta médica no dia de aplicação do questionário.

Para comparação das áreas urbano e rural, foram calculadas as significâncias estatísticas, considerando-se o teste de qui-quadrado e o teste de Fisher, úteis no caso de estratificações de pequenas amostras e supondo-se que as mesmas pudessem ser consideradas como amostras aleatórias independentes. Existe uma limitação na sel eção e na validade externa das amostras no que se refere à forma de coleta dos dados que considerou, após a identificação da usuária selecionada, entrevistas realizadas ora no domicílio, ora na própria unidade de saúde. Por esse motivo, também optamos por não realizar a expansão da amostra.

A atenção destinada às crianças de 0-5 anos, uma das principais clientelas do PSF, foi avaliada a partir das informações prestadas pelos responsáveis principais ou pelos acompanhantes das consultas. As respostas foram registradas em questionários conten do perguntas em sua maioria fechadas. A fase de coleta de dados teve início 
com a apresentação e discussão dos objetivos da pesquisa e do questionário com a equipe de entrevistadores (alunos dos cursos de medicina e enfermagem da FESO) e a coordenação da Estratégia de Saúde da Família. Para validação da formulação e compreensão do questionário, foi realizado, em seguida, pré-teste na Unidade Beira Linha. Por fim, o instrumento foi revisado e sua versão final distribuída para aplicação durante uma semana típica de atendimento.

A aplicação do instrumento combinou duas estratégias: (i) a ida aos domicílios durante as visitas domiciliares, acompanhando um dos agentes comunitários de cada equipe, (ii) a abordagem na própria unidade de saúde após 0 atendimento. A entrevista a usuários diretamente nos serviços de saúde ou em domicílios assistidos tem sido utilizada em estudos sobre atenção primária ${ }^{11,12}$ como forma de promover avaliações rápidas de caráter exploratório, embora deixem de cobrir a população que não acessou a estes serviços, seja por barreiras diretas ou por desinteresse.

Em nosso estudo, os responsáveis responderam a um questionário fechado aplicado em domicílio por meio de visita indicada pelos agentes desaúdeou no próprio módulo de atendimento. Para a avaliação da percepção de qualidade, foram apresentadas escalas de zero a dez sobre 0 último atendimento ou contato realizado por médicos, enfermeiros, auxiliares de enfermagem, agentes de saúde e pelo serviço em geral. Estes resultados foram categorizados em uma escala de cinco intervalos com variação entre muito ruim a muito bom.

\section{Resultadoseanálise}

Os respondentes foram os responsáveis pelas crianças, definidos como acompanhantes das consultas. O bserva-se uma diferença significativa em relação ao sexo dos responsáveis. Como esperado, a maioria é formada por mulheres $(91,1 \%)$. Verifica-seainda que a presença masculina é maior nas áreas rurais $(11,7 \%)$ do que nas urbanas $(6,7 \%)$. Tal fato pode ser devido ao trabalho rural e a maior permanência, em termos proporcionais, de homens em casa do que nas áreas urbanas. Outro aspecto é que a grande maioria dos responsáveis acompanhou a consulta anterior (90,9\%), sugerindo vínculos estáveis com a criança e com a rotina do serviço de saúde. N este caso, as diferenças entre as áreas não foram significativas. 0 vínculo do informante e responsável com o serviço parece elevado, pois a maioria $(90,2 \%)$ possui cadastro no PSF, sendo esta participação proporcional significativamente maior na área rural. Com relação ao grau de parentesco, a mãe representa o vínculo mais frequente $(71,6 \%)$ e as relações familiares tradicionais predominam fortemente sobre outros vínculos (2,2\%). As diferenças segundo área não foram significativas. 0 grau de escolaridade dos responsáveis revela diferenças significativas entre as áreas rurais e urbanas, sendo a escolarização maior nas áreas urbanas. De qualquer forma, predomina em ambos os grupos a escolarização em nível de primeiro grau incompleto, maior na área rural $(80,1 \%)$ do que na urbana $(58,8 \%)$.

Com relação às crianças, há predomínio de meninas (54,1\%) sobre meninos (45,9\%), não sendo estas diferenças estatisticamente significativas. $M$ ais significativas (marginalmente a $p=0,0867$ ) são àquelas relativas à frequência à creche ou escola, que é maior na área urbana $(21,9)$ do que na rural $(16,3)$. N o entanto, o mais importante aqui é o fato de que a maioria das crianças não frequenta creche ou escola $(80,6 \%)$. Embora este percentual possa ser explicado pela manutenção do cuidado em casa, pode, ao mesmo tempo, expressar a ausência de boas creches públicas na região que assegurem o acesso a préescola de qualidade para estas crianças.

A vinculação com instituições públicas de aten ção e cuidado é evidenciada de modo intenso pela posse do Cartão da Criança pela quase totalidade dos observados (94,6\%) eeste vínculo é ainda significativamente maior na área rural (98,1\% a 91,8\%). Isto pode sugerir uma vinculação maior destas populações ao programa. Perguntados sobre o estado de saúde das crianças, foi considerado "muito bom" para $27,6 \%$ dos informantes e "bom" para $60,9 \%$. As diferenças entre as áreas são bastante significativas $(p<0,0001)$ quando observadas para a condição "muito bom", melhor paraa áreaurbana (39,1\%) que para a rural $(13,3 \%)$.

As condições de acesso aos serviços podem ser avaliadas segundo os meios de locomoção utilizados pelos usuários (Tabela 1). A grande maioria dos usuários foi ao posto de saúde a pé $(85,4 \%)$. Isto sugere que estas unidades estão próximas das moradias das pessoas, o que é esperado no caso do PSF, um programa com focalização de clientela. No entanto, observam-se diferenças significativas entre as áreas. Enquanto na área urbana $96,4 \%$ dos usuários vai a pé aos serviços, na área rural, este percentual cai para $71,6 \%$. Em áreas rurais, o uso de ônibus é de magnitude importante $(18,6 \%)$, em virtude da 
maior dispersão dos domicílios, o que torna os módulos mais próximos de al guns domicílios do que de outros.

Outro meio de avaliar o acesso aos serviços é dado pelo tempo médio gasto para chegar ao seu destino. Aqui se confirma que as caminhadas até as unidades de saúde eram de curta duração, em sua maior parte: $62,2 \%$ dos usuários gastaram atédez minutos, independentedo meio de locomoção para chegar aos módulos e um número menor $(16,1 \%)$ gastou mais de vinte minutos. Seguindo a tendência observada, aqui também as diferenças entre as áreas foram significativas e os moradores em área urbana apresentaram, proporcionalmente, maior facilidade de acesso aos serviços (70,9\% gastaram menos de dez minutos, contra $49,8 \%$ dos moradores em áreas rurais).

Tabela 1. Características selecionadas, segundo situação de domicílio e informações de responsáveis, PSF de Teresópolis (RJ), 2005.

\begin{tabular}{|c|c|c|c|}
\hline & Rural & Urbano & Total \\
\hline \multicolumn{4}{|c|}{ M eio de locomoção $(p<0,0001)$} \\
\hline À pé & $189(71,6)$ & $318(96,4)$ & $507(85,4)$ \\
\hline De ônibus & $49(18,6)$ & $7(2.1)$ & $56(9,4)$ \\
\hline De carro & $19(7,2)$ & $5(1.5)$ & $24(4,0)$ \\
\hline Outros & $7(2,7)$ & $0(0,0)$ & $7(1,2)$ \\
\hline Total & $264(100,0)$ & $330(100,0)$ & $594(100,0)$ \\
\hline \multicolumn{4}{|c|}{ Tempo para chegar à unidade $(p<0,0001)$} \\
\hline Até 10 minutos & $101(49,8)$ & $205(70,9)$ & $306(62,2)$ \\
\hline De 11 a 20 minutos & $45(22,2)$ & $62(21,5)$ & $107(21,8)$ \\
\hline Mais de 20 minutos & $57(28,1)$ & $22(7,6)$ & $79(16,1)$ \\
\hline Total & $203(100,0)$ & $289(100,0)$ & 492 a $(100,0)$ \\
\hline \multicolumn{4}{|c|}{ Consultas médicas, último ano ( $p=0,0019)$} \\
\hline Nenhuma & $23(9,0)$ & $27(8,4)$ & $50(8,7)$ \\
\hline Uma a três & $110(42,8)$ & $118(36,8)$ & $228(39,5)$ \\
\hline Quatro a seis & $82(31,9)$ & $80(24,9)$ & $162(28,0)$ \\
\hline Sete ou mais & $42(16,3)$ & $96(29,9)$ & $138(23,9)$ \\
\hline Total & $257(100,0)$ & $321(100,0)$ & $578^{b}(100,0)$ \\
\hline \multicolumn{4}{|c|}{ Visitas domiciliares, último ano ( $p=0,3137)$} \\
\hline Nenhuma & $72(29,6)$ & $117(36,5)$ & $189(33,5)$ \\
\hline Uma a três & $68(28,0)$ & $74(23,1)$ & $142(25,2)$ \\
\hline Quatro a seis & $36(14,8)$ & $42(13,1)$ & $78(13,8)$ \\
\hline Sete ou mais & $67(27,6)$ & $88(27,4)$ & $155(27,5)$ \\
\hline Total & $243(100,0)$ & $321(100,0)$ & $564^{c}(100,0)$ \\
\hline \multicolumn{4}{|c|}{$\begin{array}{l}\text { Realização de exames solicitados pelos médicos } \\
(p=0,6905)\end{array}$} \\
\hline Sim & $40(15,4)$ & $43(14,2)$ & $83(14,7)$ \\
\hline Não & $220(84,6)$ & $260(85,8)$ & $480(85,3)$ \\
\hline Total & $260(100,0)$ & $303(100,0)$ & $563^{d}(100,0)$ \\
\hline \multicolumn{4}{|c|}{$\begin{array}{l}\text { As consultas são agendadas pela unidade de saúde? } \\
(p=0,8663)\end{array}$} \\
\hline Sim & $176(67,4)$ & $224(68,1)$ & $400(67,8)$ \\
\hline Não & $85(32,6)$ & $105(31,9)$ & $190(32,2)$ \\
\hline Total & $261(100,0)$ & $329(100,0)$ & $590^{e}(100,0)$ \\
\hline \multicolumn{4}{|c|}{$\begin{array}{l}\text { Responsáveis já utilizaram o mesmo serviço? } \\
(p=0,7108)\end{array}$} \\
\hline Sim & $179(67,8)$ & $219(66,4)$ & $398(67,0)$ \\
\hline Não & $85(32,2)$ & $111(33,6)$ & $196(33,0)$ \\
\hline Total & $264(100,0)$ & $330(100,0)$ & $594(100,0)$ \\
\hline
\end{tabular}

Fonte: Trabalho de campo realizado nas áreas cobertas pela Estratégia de Saúde da Família do município de Teresópolis (RJ) no ano de 2004.

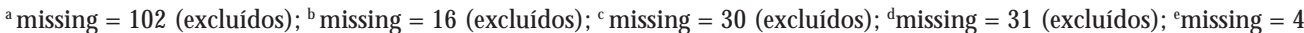
(excluídos); $p$-valor pelo teste do qui-quadrado/teste de Fisher. 
Com relação ao uso regular de serviços, as estratégias de saúde da família envolvem duas rotinas básicas. Uma delas é a consulta com mé dicos generalistas no próprio módulo de Saúde da Família e a outra se refere a visitas domiciliares periódicas realizadas por moradores treinados em programas de saúde pública (denominados agentes comunitários de saúde).

Com relação às consultas, os dados da Tabela 1 mostram que apenas $8,7 \%$ dos responsáveis relataram que a criança não foi consultada pelo médico no último ano, o que mostra que a grandemaioria frequentou o médico pelo menosuma vez ao ano. Em contrapartida, chama a aten ção 0 relato de $33,5 \%$ dos responsáveis dequenão receberam nenhuma visita domiciliar neste período e, especialmente, a significativa diferença ( $36,5 \%$ a $29,6 \%$ ) observada nas áreas urbanas erurais, tendo em vista que a rotina de visitas é considerada elemento-chave na estratégia de saúde da família.

Com relação ao acesso aos serviços de saúde, os dados na Tabela 1 não apresentam diferenças significativas entre as áreas. Ao mesmo tempo, mostram barreiras no módulo ao acesso a exames solicitados pelos médicos; por exemplo, $85,3 \%$ dos entrevistados informaram que estes pedidos não foram realizados. Outro dado que chama a atenção é o do número de consultas realizadas sem agendamento, em torno de $80,6 \%$ do total, o que pode sugerir fal has na rotina de acompanhamento das crianças, na medida em que os protocolos preconizam a realização de consultas sistemáticas nesta faixa de idade. Outra possibilidade é a grande disponibilidade de horários médicos ou de enfermagem terem tornado o agendamento desnecessário. No entanto, estes resultados analisados em conjunto com outros (como a baixa cobertura de visitas domiciliares) sugerem que os protocolos mais rígidos da programação em saúde (uma das principais ferramentas do PSF) não estejam em pleno uso no município. Apesar disso, o contingente de entrevistados que já utilizaram os serviços do módulo érelativamente alto (67\%) o que mostra adesão da população ao serviço.

Quanto ao acesso aos medicamentos, 44,3\% dos entrevistados afirmam receber gratuitamente os remédios prescritos no posto e apenas $35,4 \%$ relatam a falta eventual destes remédios. Estes resultados podem ser considerados satisfatórios, desde que se considere que 0 acesso a medicamentos seja um problema estrutural do sistema público no país. 0 acesso aos medicamentos no meio urbano foi significativamentemelhor queno rural.
Um dos principais problemas de acesso aos serviços de saúde se observa em condições agudas. Com relação a crianças, a existência de febre ou diarréia exige especial atenção. $\mathrm{Na}$ Tabela 2, se observa que as diferenças entre as áreas urbana e rural na procura dos serviços de saúde foram significativas e que $50,1 \%$ dos responsáveis dizem tratar em casa destas condições clínicas de risco e que apenas 32,5\% buscam a unidade do PSF para este primeiro atendimento. As restrições de acesso (ou baixa motivação) se mostram mais presentes entre os moradores de áreas rurais.

Embora os serviços do PSF envolvam um bom contingente de usuários, os dados até aqui analisados têm mostrado que uma parcela importante da população adscrita fica à margem de suas rotinas. A estratégia do PSF éde proteção social ampla, em quetodos os moradores da área configuram o público-alvo. Além disso, o programa representaria a porta de entrada dos moradores ao SUS. No entanto, na Tabela 2, observa-se que para $44,6 \%$ dos responsáveis a ida ao posto não ocorre por rotina (como para outros $46,6 \%$ ), mas apenas em caso de doença ou malestar (ou seja, em condições agudas). Cabe destacar que o uso em condições agudas é maior nas áreas rurais do que nas áreas urbanas, o que pode sinalizar a maior facilidade de acesso a serviços de emergência nas áreas urbanas, ao passo que nas áreas rurais estes não existem.

O vínculo dos usuários aos serviços do PSF pode ser medido pelo modo como recebem informações relevantes pelos profissionais de saúde ou como apreendem as informações prestadas. Na Tabela 2, se nota que 83,5\% dos entrevistados conhecem o nome do médico que realizou a última consulta. As diferenças entre as áreas foram marginalmente significativas e, neste caso, a adesão de informantes de áreas rurais foi maior (85,6\% a 80,0\%). Estes dados sugerem uma vinculação positiva dos usuários aos serviços. I gualmente, $88,1 \%$ conhecem o motivo da consulta e suas implicações gerais. Reforçando as observações em tabelas anteriores, dentre os que conhecem os motivos da consulta realizada (um total de 512 informantes), para $72,3 \%$ ela ocorreu para tratamento de condições clínicas específicas. Isto sugere que as rotinas nos moldes da agenda da promoção da saúde (que implicam o uso dos serviços de saúde fora de condições agudas) ainda não são predominantes nestes serviços.

Os dados apresentados na Tabela 3 sugerem uma boa aceitação e satisfação dos usuários com os serviços de saúde. 
Tabela 2. Procedimento principal do acompanhante em caso de febre ou diarréia da criança, principal condição em que o responsável leva a criança ao módulo do PSF e nível de conhecimento sobre atendimentos realizados, segundo situação de domicílio e informações de responsáveis, PSF de Teresópolis (RJ)

\begin{tabular}{|c|c|c|c|}
\hline & Rural & Urbano & Total \\
\hline \multicolumn{4}{|l|}{ Procedimento frente a sintomas $(p=0,0090)$} \\
\hline Trata em casa & $148(56,5)$ & $145(44,9)$ & $293(50,1)$ \\
\hline Procura centro de saúde & $7(2,7)$ & $14(4,3)$ & $21(3,6)$ \\
\hline Procura unidade básica de saúde & $68(26,0)$ & $122(37,8)$ & $190(32,5)$ \\
\hline Procura agente comunitário de saúde & $0(0,0)$ & $2(0,6)$ & $2(0,3)$ \\
\hline Procura hospital & $39(14,9)$ & $40(12,4)$ & $79(13,5)$ \\
\hline Total & $262(44,8)$ & $323(55,2)$ & $585^{\mathrm{a}}(100,0)$ \\
\hline \multicolumn{4}{|l|}{ Condição para busca por atendimento $(p=0,0003)$} \\
\hline Apenas em caso de doença ou mal estar & $139(52,7)$ & $126(38,2)$ & $293(44,6)$ \\
\hline Em consultas agendadas & $97(36,7)$ & $180(54,6)$ & $277(46,6)$ \\
\hline Apenas por encaminhamento de visitador & $3(1,1)$ & $2(0,6)$ & $5(0,8)$ \\
\hline Outros & $25(9,5)$ & $22(6,7)$ & $47(7,9)$ \\
\hline Total & $264(44,4)$ & $330(55,6)$ & $594(100,0)$ \\
\hline \multicolumn{4}{|l|}{ Consultas médicas, último ano $(p=0,0019)$} \\
\hline Nenhuma & $23(9,0)$ & $27(8,4)$ & $50(8,7)$ \\
\hline Uma a três & $110(42,8)$ & $118(36,8)$ & $228(39,5)$ \\
\hline Quatro a seis & $82(31,9)$ & $80(24,9)$ & $162(28,0)$ \\
\hline Sete ou mais & $42(16,3)$ & $96(29,9)$ & $138(23,9)$ \\
\hline Total & $257(100,0)$ & $321(100,0)$ & $578^{b}(100,0)$ \\
\hline \multicolumn{4}{|c|}{$\begin{array}{l}\text { Conhece o nome do médico que realizou a última } \\
\text { consulta? }(p=0,0743)\end{array}$} \\
\hline Sim & $226(85,6)$ & $264(80,0)$ & $490(83,5)$ \\
\hline Não & $38(14,4)$ & $66(20,0)$ & $104(17,5)$ \\
\hline Total & $264(100,0)$ & $330(100,0)$ & $594(100,0)$ \\
\hline \multicolumn{4}{|l|}{$\begin{array}{l}\text { Conhece o motivo informado pelo médico para a } \\
\text { última consulta? }(p=0,1790)\end{array}$} \\
\hline Sim & $216(86,1)$ & $296(89,7)$ & $512(88,1)$ \\
\hline Não & $35(13,9)$ & $34(10,3)$ & $69(11,9)$ \\
\hline Total & $251(100,0)$ & $330(100,0)$ & $581^{c}(100,0)$ \\
\hline \multicolumn{4}{|c|}{$\begin{array}{l}\text { M otivo da consulta, entre os que souberam informa? } \\
n=512 ;(p=0,0015)\end{array}$} \\
\hline Tratamento & $172(79,3)$ & $198(66,9)$ & $370(72,3)$ \\
\hline Rotina & $44(20,4)$ & $98(33,1)$ & $142(27,7)$ \\
\hline Total & $216(100,0)$ & $296(100,0)$ & $512(100,0)$ \\
\hline
\end{tabular}

Fonte: Trabalho de campo realizado nas áreas cobertas pela Estratégia de Saúde da Família do município de Teresópolis (RJ) no ano de 2004.

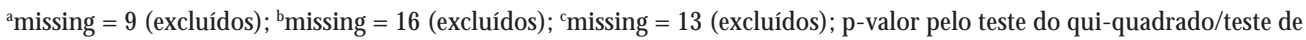
Fisher.

Tabela 3. O pinião sobre atendimento prestado por profissionais de saúde, segundo situação de domicílio e informações de responsáveis, PSF de Teresópolis (RJ), 2005.

\begin{tabular}{llrrr}
\hline \multicolumn{1}{c}{ Profissionais } & Qualificações & \multicolumn{1}{c}{ Rural } & Urbano & \multicolumn{1}{c}{ Total } \\
\hline M édicos $(p=0,0064)$ & Muito bom ${ }^{\text {a }}$ & $189(75,0)$ & $267(86,1)$ & $456(81,1)$ \\
& Total & $252(100,0)$ & $310(100,0)$ & $562^{b}(100,0)$ \\
Agentes comunitários $(p=0,4789)$ & Muito bom ${ }^{\text {a }}$ & $199(80,2)$ & $248(86,8)$ & $448(83,7)$ \\
& Total & $248(100,0)$ & $287(100,0)$ & $55^{c}(100,0)$
\end{tabular}

${ }^{\text {a }}$ muito bom representado por notas 9 ou 10 em escala de $0-10 ;{ }^{b}$ missing $=32$ (excluídos) $;{ }^{c}$ missing $=32$ (excluídos). 
Para 81,1\% dos entrevistados, 0 atendimento pelos médicos foi muito bom e para $83,7 \%, 0$ serviço dos agentes de saúde foi muito bom. As diferenças entre áreas foram significati vas para 0 atendimento médico e a aprovação foi maior na área urbana. Os resultados para a qualidade do serviço como um todo e de profissionais de enfermagem seguiram a mesma tendência. Anteriormente, havíamos observado que 189 entrevistados $(33,5 \%)$ referiram não ter sido objeto de nenhuma visita domiciliar no último ano. Este número é elevado, pois estas visitas fazem parte da rotina de trabalho das equipes. Analisamos em seguida o comportamento de dois grupos. Um deles, sem relato de visita no ano, frente aos demais entrevistados (em total de 387) queregistraram ao menos uma visita domiciliar. Podemos considerar a hipótese de que o grupo não visitado seja menos aderido ou tenha menor acesso aos recursos do PSF. Na Tabela 4, observamos quea clientela não visitada tem menor acesso gratuito aos medicamentos receitados e a consultas agendadas previamente pel os serviços eque estas diferenças são marginalmente significativas. Notamos ainda que a lembrança do nome do médico ésignificativamentemaior entrea clientela que recebeu ao menos uma visita domiciliar no ano. Estes dados sugerem que a visita domiciliar representa um relevantefacilitador do acesso aos serviços e de adesão ao PSF. Com relação à percepção de qualidade de médicos e de agentes comunitários, a condição devisitado não se mostrou um fator importante de diferenciação entre os grupos estudados.

\section{Consideraçõesfinais}

0 estudo foi realizado por meio de questionários aplicados aos responsáveis pelas crianças no momento do acesso aos serviços nos módulos de saúde da família ou em visitas domiciliares. Isto facilita a avaliação rápida e a economia de recursos, porém deixa de fora populações que não acessaram os serviços de saúde. A qualidade do acesso aqui avaliada parte da experiência de usuários diretos destes serviços.

Consideramos os aspectos mais relevantes da estratégia de saúde da família no Brasil e observamos o seu comportamento na região estudada. A maior escolarização nas áreas urbanas deve ser considerada ao se avaliar as percepções distintas entre estes grupos, embora em ambos o nível de escolarização seja baixo. A vinculação preliminar da população alvo ao programa foi elevada, medida pela posse do Cartão da Criança. A percepção positiva dos informantes sobre o estado de saúde das crianças foi elevada, diferindo estes usuários do observado em programas implementados em áreas de extrema pobreza e típicas da acelerada difusão dos módulos de saúde da família no país.

As condições de acesso dos usuários foi positiva, tendo a grande maioria chegado a pée gasto menos de dez minutos no deslocamento, sugerindo a proximidade dos serviços aos locais de moradia. A grande maioria frequentou ao menos uma vez ao médico, embora em grandepartesem agendamento prévio da consulta. No entanto, cerca de um terço dos informantes relatou a au-

Tabela 4. Qualidade da atenção segundo a realização de visitas domiciliares e informações de responsáveis, PSF de Teresópolis (RJ), 2005.

\begin{tabular}{lrrrr}
\hline \multicolumn{1}{c}{ Variáveis } & Sim & Não & Às vezes & Total \\
\hline Acesso gratuito aos medicamentos receitados? $(p=0,0733)$ & & & & \\
$\quad$ Visitados & $183(47,3)$ & $71(18,3)$ & $133(34,4)$ & $387(100,0)$ \\
$\quad$ Não visitados & $71(38,0)$ & $47(24,6)$ & $70(37,4)$ & $187(100,0)$ \\
Lembra o nome do médico? ( $p<0,0001)$ & & & \\
$\quad$ Visitados & $355(87,7)$ & $50(12,3)$ & - & $405(100,0)$ \\
$\quad$ Não visitados & $135(71,4)$ & $54(28,6)$ & - & $189(100,0)$ \\
Consultas são marcadas pelo próprio serviço? $(p=0,0843)$ & & & \\
$\quad$ Visitados & $283(70,1)$ & $121(29,5)$ & - & $404(100,0)$ \\
$\quad$ Não visitados & $117(62,9)$ & $69(37,1)$ & - & $187(100,0)$
\end{tabular}

Fonte: Trabalho de campo realizado nas áreas cobertas pela Estratégia de Saúde da Família do município de Teresópolis (RJ) no ano de 2004.

p-valor pelo teste do qui-quadrado/teste deFisher. 
sênciadevisita domiciliar no último ano. Isto pode ser devido à facilidade de acesso aos módulos de saúde da família ou à fragilidade relativa nestes procedimentos, especialmente em áreas urbanas.

Um ponto crítico apontado em avaliações destes programas éo acesso aos exames eisto foi corroborado neste estudo. A grande mai oria dos entrevistados disse que os exames solicitados não foram realizados. Outro ponto crítico apontado em estudos anteriores éo acesso aos medicamentos. Neste caso, mais da metade dos usuários não recebemedicamentos gratuitamente de modo regular e constante, embora neste grupo a mai oria os receba de modo eventual, sendo a distribuição mais eficiente em áreas urbanas. Uma medida importante do acesso aos serviços está nas condições agudas. Neste estudo, as restrições de acesso foram significativamente maiores nas áreas rurais. Cerca da metade dos informantes trataram inicialmente em casa condições de risco clínico como febres e diarréias.

Com relação aos vínculos com os profissionais, a grande maioria conhece o nome do médico que realizou a última consulta, os motivos da consulta e de suas implicações. A maior parte das consultas ocorreu por queixas ou sinais clínicos e não pelo cumprimento de protocolos de prevenção. A satisfação manifestada pelos usuários quanto aos médicos, aos agentes de saúde, aos profissionais de enfermagem e ao conjunto dos serviços foi el evada. Observamos que entrea clientela não visitada por agentes de saúde no último ano houve menor acesso à distribuição de medicamentos e menor lembrança do nome do médico consultante. Isto sugere que a visita domiciliar representa facilitador do acesso aos serviços e de adesão ao PSF.

Os pontos críticos observados (baixa cobertura de visitas domiciliares, dificuldades na realização de exames e no fornecimento regular de medicamentos e o tratamento frequente de condições agudas no domicílio) sugerem que o programa, apesar de bem avaliado para 0 atendimento rotineiro de primeira linha, enfrenta importantes dificuldades em promover 0 acesso da clientela a serviços correlatos, mesmo os de baixa complexidade.

Este estudo não avaliou as dimensões de custo e de custo-efetividade do programa e isto deve ser levado em conta em avaliações futuras. Tratase deuma lacuna importante em estudos avaliativos em nosso país. M ais importante ainda, deve ser considerada a dimensão do custo de oportunidade. Embora muitas vezes bem avaliados, sabemos pouco sobre os custos de se adotar estratégias distintas em termos de gastos nominais, gastos comparados e, principalmente, na comparação de resultados e impactos de estratégias distintas. Como observamos anteriormente, os estudos comparados entre módulos de saúde da família e unidades convencionais lidam pouco com aspectos relacionados ao financiamento eao impacto dos serviços e mais com itens específicos da qualidade da atenção aos usuários.

\section{Colaboradores}

JM Ribeiro, SAV Siqueira e LFS Porto participaram igualmente de todas as etapas da elaboração do artigo. 


\section{Referências}

1. Brasil. M inistério da Saúde. Política Nacional da Atenção Básica. Série Pactos pela Saúde. Volume 4. Brasília: Ministério da Saúde; 2006.

2. Kusek JZ, Rist RC. Ten steps to a results-based monitoring and evaluation system: a handbook for development practitioners. Washington, D.C.: World Bank: 2004.

3. Szwarcwald $\mathrm{CL}$, Castilho EA. Estimativa do número de pessoas de 15 a 49 anos infectadas pelo HIV, Brasil, 1998. Cad Saude Publica 2000; 16(Supl.1):135141.

4. Caminal J, Sánchez $E$, M orales $M$, Peiró R, Márquez $\mathrm{S}$. Investigation progress in Spain with "Ambulatory Care Sensitive Conditions". Rev Esp Salud Pública 2002; 76:189-196.

5. Villanueva $\mathrm{H}$. Ongoing health management control by cost monitoring through tracer indicators. Rev Panam Salud Publica 2001; 10:405-412.

6. Kessner DM, Kalk CE, Singer JA. Assessing health quality - the case for tracers. N Eng J M ed , 1973; 288:189-194.

7. Kessner DM, Kalk CE, Singer JA. Evaluación de la calidad de la salud por el método de los procesos trazadores. In: White KL, editor. Investigaciones sobre servicios de salud: una antología. [Publicación Científica no 534]. Washington, D.C,: Organización Panamericana de la Salud; 1992. p. 555-563.

8. Hartz Z, Champagne F, Leal MC, Contandriopoulos AP. M ortalidade infantil "evitável" em duas cidades do Nordeste do Brasil: indicador de qualidade do sistema local de saúde. Rev. Saude Publica 1996; 30:310-318.

9. Donabedian A. An introduction to quality assurance in health care. New York: Oxford University Press; 2003.

10. Contandriopoulos AP. Avaliando a institucionalização da avaliação. Cien Saude Colet 2006; 1(3):705711.

11. Bodstein RC, Feliciano K, Hortale VA, Leal MC. Estudos de Linha de Base do Projeto de Expansão e Consolidação do PSF: considerações sobre o seu acompanhamento. Cien Saude Colet 2006; 1(3):725731.

12. Rehem TCM SB, Trad LAB. Assistência Domiciliar em Saúde: subsídios para um projeto de atenção básica brasileira. Cien Saude Colet 2005; 10(Sup.):231242.

13. Viana ALA, Rocha JSA, Elias PE, Ibañez N, Novaes MHD. Modelos de atenção básica nos grandes municípios paulistas: efetividade, eficácia, sustentabilidade e governabilidade. Cien Saude Colet 2006; 11(3):577-606
14. Roncalli AG, Lima KC. Impacto do PSF sobre indicadores de saúde da criança em municípios de grande porte da Região Nordeste do Brasil. Cien Saude Colet 2006; 1(3)712-724.

15. Ibanez N, Rocha JSY, Castro PC, Ribeiro MCSA, Forster AC, Novaes MHD, Viana ALA. Avaliação do Desempenho da Atenção Básica no Estado de SP. Cien Saude Colet 2006; 1(3):683-703.

16. Szwarcwald C, M endonça M H, Andrade CTA. Indicadores de Atenção Básica em quatro Municípios do Estado do Rio de Janeiro: resultado de inquérito populacional de base domiciliar. Cien Saude Colet 2006; 1(3):643-655.

17. Ribeiro JM, Costa NR, Pinto LFS, Silva PLB. Atenção ao pré-natal na percepção das usuárias do Sistema Ú nico de Saúde: um estudo comparativo. Cad Saude Publica 2004; 20(2):534-545.

18. Almeida C, Macinko J. Validação de uma metodologia de avaliação rápida das características organizacionais e do desempenho dos serviços de atenção básica do Sistema Ú nico de Saúde (SUS) em nível local. Brasília: Organização Pan-Americana de Saúde; 2006.

19. Pinheiro $Y$, Dimenstein $M$. Estrutura e organização do trabalho do cirurgião-dentista no PSF de municípios do RN. Cien Saude Colet 2006; 1(3):219-227.

20. M arques RM, M endes $A$. Atenção Básica e PSF: novos rumos para a política de saúde e financiamento. Cien Saude Colet 2003; 8(2):403-415.

21. Pereira ATS, Campelo ACFS, Cunha FS, Noronha J, Cordeiro H, Dain S, Pereira TR. Sustentabilidade Econômica-Financeira no Proesf em Municípios do Amapá, Pará, M aranhão e Tocantins. Cien Saude Colet 2006; 1(3):607-620.

22. Leavell H, Clark EG. M edicina preventiva. São PauIo: McGraw-Hill do Brasil; 1976.

23. Schraiber LB, organizador. Programação em saúde hoje. São Paulo: Hucitec; 1990.

24. Starfield B. Atenção primária: equilíbrio entre necessidades de saúde, serviços e tecnologia. Brasília: UNESCO/M inistério da Saúde; 2002.

Artigo apresentado em 22/04/2008

Aprovado em 31/10/2008

Versão final apresentada em 15/02/2009 